\begin{tabular}{|l|l|c|c|}
\hline Eiszeitalter u. Gegenwart & $\mathbf{2 9}$ & $\begin{array}{c}173-178 \\
1 \mathrm{Kt} .\end{array}$ & Hannover 1979 \\
\hline
\end{tabular}

\title{
Rinnen an der Basis des glaziären Pleistozäns in Schleswig-Holstein
}

\author{
WINFRIED HINSCH *) \\ Tertiary, Pleistocene, paleorelief, channel, glacial erosion, fluvioglacial, salinary structures \\ NW-German Lowlands, Schleswig-Holstein
}

Kurzfassung: Nach Bohrungsauswertung wird der präquartäre Untergrund und die Basis des glaziären Pleistozäns in Schleswig-Holstein dargestellt. Die Basis des Pleistozäns zeigt Hochgebiete und Rinnensysteme, deren Verlauf weitgehend unabhängig ist von den halokinetisch bedingten Trögen und Salinarstrukturen des Untergrundes. Rinnen in verschiedenen Richtungen sind relativ rasch durch exarative Glazialtektonik und subglaziäre Erosion entstanden. Für viele Rinnen läßt sich ein elstereiszeitliches Alter nachweisen.

\section{[Channels at the Base of Glacial Pleistocene in Schleswig-Holstein]}

A b s t r a ct: Using well data the prequarternary subcrop and the base of glacial pleistocene is mapped. There are elevations of subcrop and channel systems at the base of pleistocene. Their configuration is rather independent of halokinetinally induced troughs and salinary structures of the subcrop. Channels in diverse directions were formed rather quickly by exaration, glacial tectonic and subglacial erosion. For many channels an Elster age can be proved.

In halts verzeichnis

1. Einleitung

2. Präquartärer Untergrund

3. Schollen an der Quartärbasis

4. Basis des glaziären Pleistozäns

5. Verlauf der quartären Rinnensysteme

6. Entstehung der Rinnen

7. Alter der Rinnen

8. Schriftenverzeichnis

\section{Einleitung}

Im Rahmen des DFG-Programms „Nordwestdeutsches Tertiärbecken“ wurden in Tiefenlinien- und Isopachenplänen die Abschnitte des Tertiärs dargestellt. Die beiden das Quartär betreffenden Karten „Basis des glaziären Pleistozäns“ (HiNscH 1977b) und „Karte des präquartären Untergrundes in Schleswig-Holstein“ (HiNscH 1997a) sind der Abschluß dieses Kartenwerkes über das Tertiär im Untergrund von Schleswig-Holstein zum Hangenden hin.

Grundlage für diese Karten ist die bio- und lithostratigraphische Auswertung der Bohrungen, deren Schichtenverzeichnisse im Bohrarchiv des Geologischen Landesamtes gespeichert sind. Es wurden alle Bohrungen ausgewertet, die das Quartär durchteuft oder

*) Anschrift des Verfassers: Dr. W. Hin s ch, Geologisches Landesamt Schleswig-Holstein, Mercatorstraße 7, D-2300 Kiel-Wik. 
mehr als $50 \mathrm{~m}$ Teufe erreicht haben. Neben den Erdölbohrungen sind dies vor allem hydrogeologische Aufschlußbohrungen. An dieser Stelle sei allen Bohrungsbearbeitern gedankt, deren Ergebnisse viel zu dem Kartenwerk beigetragen haben. Auf top. Karten $1: 25000$ wurden die Bohrungen eingetragen und die Basis des glaziären Pleistozäns auf NN bezogene Werte umgerechnet. Die sich hieraus ergebenden Tiefenlinien und Ausbisse wurden auf Úbersichtskarten im Maßstab $1: 250000$ übertragen.

Der vorliegenden Arbeit ist eine Karte (Kt. 1) im Maßstab 1:500000 beigegeben, auf der in einer durch den Maßstab gebotenen vereinfachten Form Salinarstrukturen, Tertiärtröge und glaziäre Rinnen in Schleswig-Holstein dargestellt sind. Die Position der Salzstrukturen ist der Arbeit von JARITZ (1973) über die Entstehung der Salzstrukturen Nordwestdeutschlands entnommen.

\section{Präquartärer Untergrund}

Als präquartärer Untergrund wurden folgende Ausbisse auskartiert: Perm, Oberkreide, Untereozän, Mitteleozän bis Oligozän, Unterer Glimmerton, marines Hemmoorium, Braunkohlensande, Oberer Glimmerton und Kaolinsande. Die Ausstriche zeigen eine starke Abhängigkeit vom salinartektonischen Bau des Untergrundes, während Anomalien dieser Ausbisse meist durch glaziäre Rinnen bedingt sind.

In einer zentralen Region alter Diapire bei Rendsburg sind die Tertiärtröge nur noch mäßig eingesenkt. Ausgehend vom flachen und breiten Rendsburger Trog nimmt beidseitig sowohl nach W zum westholsteinischen Troggebiet als auch nach E zum „Hamburger Loch“ (Pagensander bis Oldesloer Trog) der Tiefgang und die Tertiärmächtigkeit der Tröge zu. Dabei schwellen die Mächtigkeiten des Alttertiärs bis zum Friedrichstädter und Kaltenkirchener Trog an, die des Jungtertiärs noch distaler zur zentralen Schwelle bis zum Gardinger und Oldesloer Trog hin an.

In den Senkungsgebieten mit besonders mächtigen Tertiärtrögen sind die Diapire von Oldenswort, Elmshorn, Quickborn, Sievershütten, Sülfeld und Segeberg bis an die Quartärbasis hochgedrungen, während bei den alten Diapiren in Mittelholstein die quartäre Erosion nur bis an die Oberkreide hinabreicht. Die Verbreitung der jüngsten lithostratigraphischen Einheit des Tertiärs, des Kaolinsandes, ist auf folgende jüngere Senkungsgebiete (Kt. 1) beschränkt:

a) Westholstein-Loch Gardinger Trog (Absenkung der Kaolinsandbasis bis $1200 \mathrm{~m}$ )

Friedrichstädter Trog

Trischener Trog

b) Hamburger Loch Wahlstedter Trog

Oldesloer Trog (Absenkung des Kaolinsandes tiefer als $500 \mathrm{~m}$ )

Nordteil des Ahrensburger Trogs

Kaltenkirchener Trog

Pinneberger Trog

Horster Trog

Pagensander Trog

c) Westteil der Westschleswig Scholle (Sylt, Amrum, Föhr)

d) Tarper Trog zwischen Westschleswig-Scholle und Angelner Schwelle.

Die fluviatilen pliozänen bis ältestpleistozänen Kaolinsande sind ursprünglich flächenhaft abgelagert worden, jedoch nur in den Gebieten mit starken Senkungstendenzen im jüngeren Tertiär erhalten geblieben. In diesem Sinne gab es keine pliozänen Täler, die als Vorläufer der glaziären Rinnen in Betracht kommen. 


\section{Schollen an der Quartärbasis}

Ein schwieriges Problem sind die glazialtektonisch gestauchten oder verschleppten Schollen und Schuppen an der Basis des glaziären Pleistozäns. Hier soll zwischen parautochthonen (fast anstehenden) Schollen, scheinbaren oder Pseudoschollen und allochthonen (offensichtlichen) Schollen unterschieden werden, von denen die ersten beiden dem präquartären Untergrund, die letzteren dem glaziären Pleistozän zugerechnet werden.

$\mathrm{Zu}$ den parautochthonen Schollen gehören zunächst Stauchendmoränen, wo das Anstehende nur in sich verschuppt ist (Beispiel Morsum-Kliff/Sylt). Ferner gehören hierher durch Pleistozänkeile nur unvollständig vom Anstehenden gelöste Schollen. Ein gutes Beispiel hierfür ist in der Bohrung Schönberg (am Westrand des Salzkissens von Nusse) eine $50 \mathrm{~m}$ mächtige Glimmertonscholle, die nach Biostratigraphie und Höhenlage noch als Fortsetzung des Anstehenden zu erkennen ist, obwohl sie durch einen $9 \mathrm{~m}$ mächtigen Pleistozänkeil (oben Geschiebemergel, unten Geschiebekies und Harnische) vom Liegenden getrennt ist (HINSCH 1975).

Bei nicht gekernten Bohrungen sind parautochthone Schollen schwer zu unterscheiden von Pseudoschollen. Diese werden vorgetäuscht durch erneut auftretenden Spätnachfall, meist auffallend gleichkörniger Feinkies, aus jüngeren Schichten lange nach Abklingen des normalen Nachfalls. Hierfür besonders prädestiniert sind feste Bänke oder Konkretionslagen wie Reinbeker Gestein und Holsteiner Gestein, bei denen das Bohrgestänge plötzlich stark gegen die Bohrlochwandung schlägt. Auch innerhalb des anstehenden Miozäns läßt sich solcher Spätnachfall nachweisen. Allochthone Schollen sind beispielsweise Eozänschollen in Gebieten mit präquartär anstehendem Miozän. Hierher gehören auch Miozänschollen in den glaziären Rinnen, wenn deren Teufenlage vom Anstehenden beträchtlich abweicht. Ein Beispiel hierfür ist in der Bohrung Meilsdorf (westlich Siek) eine Scholle von Tostedter Schichten, die ca. $60 \mathrm{~m}$ tiefer als das Anstehende im Niveau des Hamburger Tons angetroffen wird (HiNsch 1975).

Aus praktischen Gründen, die sich aus Nachfallmöglichkeiten während des Bohrvorganges ergeben, bleibt nichts anderes übrig, als die meist nicht unterscheidbaren parautochthonen Schollen und Pseudoschollen dem präquartären Untergrund zuzurechnen, während die eindeutig allochthonen Schollen dem Pleistozän zugeordnet werden. Die tatsächliche Eindringtiefe der Glazialtektonik in den Untergrund muß bei den gegenwärtig vorwiegenden Spülbohrverfahren als weitgehend hypothetisch gelten. Man kann jedoch annehmen, daß mit zunehmender Tiefe die Sprungbeträge bald unbedeutend werden.

\section{Basis des glaziären Pleistozäns}

Nach den Grundsätzen des vorigen Kapitels wird auf der 1977 im Geologischen Landesamt Schleswig-Holstein erschienenen Karte die Basis des glaziären Quartärs in folgenden Tiefenstufen dargestellt: höher als NN, $0-25 \mathrm{~m}, 25-50 \mathrm{~m}, 50-100 \mathrm{~m}, 100-200 \mathrm{~m}$ und tiefer als $200 \mathrm{~m}$. Die Karte der Quartärbasis konnte im N an die Abbildung der Quartärbasis durch RAsmussen (1966) angeschlossen werden. Im S war eine Konnektierung mit den Rinnen in Nordniedersachsen (Kuster \& MEYER 1979, in diesem Band) möglich, während für Südostholstein die von JohanNSEN \& Nachtigali (1972) beschriebenen Rinnen bestätigt werden konnten. Der Rinnenverlauf im Hamburger Raum konnte den Arbeiten von KocH (1924) und LöHNERT (1966) entnommen werden.

Ein wesentlicher Teil meiner Arbeit ist die Darstellung in Tiefenstufen. Aus drucktechnischen Gründen konnten in Kt. 1 nur die Rinnen mit Tiefen unter $-100 \mathrm{~m} \mathrm{NN}$ dargestellt werden. Jedoch ist auch hieran zu erkennen, daß die im ganzen Tertiär vorhandene starke Abhängigkeit der Tiefenlage und Mächtigkeit der Schichtglieder von der 
Salinartektonik, die sich in breiten Trögen und Mulden zwischen den Salinaren und verstärkter Salzabwanderung zu den jungen Diapiren zeigt, an der Basis des glaziären Pleistozäns nicht mehr vorhanden ist. Die sich abzeichnenden Rinnen sind wesentlich schmaler und kleinräumiger als die Mulden und Tröge des Tertiärs und zeigen meist auch andere Richtungen als diese.

Die tiefsten Stellen der Quartärbasis wurden bei Hamburg in der Ellerbeker Rinne westlich von Schnelsen mit $322 \mathrm{~m}$ unter NN und bei Tönning an der Eidermündung mit $350 \mathrm{~m}$ unter NN angetroffen. Eine gewisse Häufung von besonders tiefen Rinnenbildungen mit Tiefen von mehr als $200 \mathrm{~m}$ zeigen die tiefen Tröge im Hamburger und Westholsteiner Raum. Eine Ausnahme von dieser Regel ist die von Lauenburg in Richtung Lübeck verlaufende Rinne, die in einem Gebiet schwacher tertiärer Absenkung liegt. Östlich von Segeberg ist die Einmuldung im Pliozän besonders stark und trotzdem läßt sich keine glaziäre Rinne nachweisen.

Größere Gebiete mit Hochlagen des Präquartärs über NN finden sich im NW bei Sylt und im SE zwischen den Rinnen im Gebiet der Trittauer Mulde und südöstlich der Ahrensburger Mulde.

\section{Verlauf der glaziären Rinnensysteme}

Aus dem Vergleich der Tiefenlinien der Tertiärhorizonte (Muldentyp) mit dem grundsätzlich anderen Verlauf der Basis des glaziären Pleistozäns (Rinnentyp) läßt sich schließen, daß die glaziären Rinnen in erster Linie durch exogene Glazialtektonik, Exaration und subglaziäre Erosion unter dem Inlandeis entstanden sind und allenfalls untergeorćnet durch den Bau des Untergrundes in Verlauf und Tiefe modifiziert wurden.

Die Unabhängigkeit der glaziären Rinnen von den Salinarstrukturen des Untergrundes zeigt sich darin, daß auch im halokinetisch ruhigen Gebiet der Westschleswig-Scholle südlich von Föhr und an der deutsch-dänischen Grenze solche Rinnen vorhanden sind. Relativ isoliert erscheinen die Rinne in der Flensburger Innenförde und im Tarper Trog (Frörup), während die Rinnen im S der Angelner Schwelle (Böklund und Süderbrarup) mit einer Rinne an der Schlei bei Schleswig im Zusammenhang stehen könnten. Rinnen mit vorwiegender W-E-Richtung finden sich bevorzugt im Eidergebiet, wo rheinisch streichende Strukturen an der Linie Husum-Kiel enden. So verlaufen Rinnen von Husum über Schleswig-Eckernförde zur Kieler Förde, vom Friedrichstädter Trog nach Rendsburg und von der Eidermündung zum Delver Trog. Weiter im S finden sich Rinnen mit dieser Richtung bei Bramstedt und Eisendorf.

Bei der Angabe der Rinnenrichtung ist zu berücksichtigen, daß sie nicht geradlinig, sondern leicht geschlängelt sind. Die Rinnen sind manchmal nur $1 \mathrm{~km}$ breit, was sich besonders in dicht abgebohrten Gebieten nachweisen läßt. Generell sind auf der vorliegenden Karte (Kt. 1) die meisten Rinnen wegen Mangel an Bohrungen eher zu breit als zu schmal dargestellt.

Etwa rheinisch streichende Rinnen folgen manchmal mit wechselndem Abstand den Salzstrukturen von Heide-Hennstedt oder Muldenachsen des Preetzer Trogs, der Hemmelsdorfer Mulde und des Oldesloer Trogs (dort eine senkrecht dazu gerichtete Abzweigung am Südende). Die Meilsdorfer Rinne biegt vom Nordteil der Sieker Struktur in rheinische Richtung um. Die auffällige rheinisch streichende Rinne von Lauenburg-Mölln liegt überwiegend im Salzkissengebiet von Nusse-Gudow.

Sehr auffällig sind NNW-SSE verlaufende, d. h. etwa eggisch streichende, glaziäre Rinnen. Diese Richtung kommt bei den Salinarstrukturen in Schleswig-Holstein sehr selten vor, so daß Ursachen, die im glaziären Pleistozän selbst liegen, zur Deutung heran- 
gezogen werden müssen. Diese Rinnen liegen am ehesten in der Richtung des Vorfluters in der nördlichen Nordsee. Allerdings haben die Rinnen kein durchgehendes Gefälle, was z. T. durch eine spätere Übertiefung im Gebiet der tiefen Tröge des Hamburger Lochs und Westholstein-Lochs verursacht sein kann. Hierzu gehört eine Rinne vom Pinneberger Trog zur Wilsterer Mulde und nach Oldenbüttel, die Ellerbeker Rinne von Hamburg nach Rendsburg und die Duvenstedter Rinne vom Ahrensburger Trog in Richtung Bramstedt. Besonders im Südteil sind diese Rinnen gut durch Bohrungen belegt. Die Ellerbeker Rinne überquert zweimal eine Salinarstruktur, nämlich den Salzstock Schnelsen und die Struktur Peissen-Gnutz. Relativ schmale glaziäre Rinnen mit vorwiegender SW-NERichtung sind die Etzer Rinne im Pinneberger Trog, die Elmshorner Rinne, die Rinne bei Hohenhorn und die Bille-Rinne von Hamburg über Witzhave zur Trittauer Mulde.

\section{Entstehung der Rinnen}

Vergleicht man die Basis des glaziären Pleistozäns mit den Tiefenlinienplänen von Tertiärhorizonten, fällt die wesentlich geringere Abhängigkeit von der Salinartektonik auf. $\mathrm{Da}$ die Salinare sich auch im Pleistozän weiter bewegt haben, können hierfür zwei Erklärungen angeboten werden.

1. Die glaziären Rinnen haben sich in wesentlich kürzerer Zeit gebildet als für die Ablagerung einer Tertiärstufe erforderlich ist. Während ein Zeitraum von 106-7 Jahren für die Ablagerung eines abgrenzbaren Tertiärabschnittes zur Verfügung steht, haben sich die glaziären Rinnen in einem Zeitraum von nur 103-4 Jahren gebildet. Bei gleicher Aktivität der Salinare ist daher der Einfluß auf die Rinnenbildung zu gering, um ihren Verlauf entscheidend zu bestimmen.

2. Die glaziären Sedimente entfalten durch Exaration und subglaziäre Erosion eine wesentlich stärkere Eigendynamik als die Sedimentation des marinen und fluviatilen Tertiärs und auch des marinen Quartärs im Nordseebecken, das sich in seiner Mächtigkeitsentwicklung und mit transgressiven Schichtausfällen sehr viel mehr und eher passiv den salinartektonisch verursachten Strukturen anpaßte.

Im einzelnen könnten die glaziären Rinnen durch Salzstockbewegungen beeinflußt werden. Sehr viel häufiger ist jedoch, daß Anomalien im Ausbiß durch glaziäre Rinnen verursacht werden. Zum Beispiel haben sich die Duvenstedter und Ellerbeker Rinne im Hamburger Raum durch den oberen Glimmerton in die Braunkohlensande eingeschnitten. Andere Rinnen haben sich durch die Braunkohlensande hindurch in das Vierlandium oder Oligozän eingetieft. Auf den Strukturen Sülfeld und Oldenswort fallen relativ breite Depressionen des glaziären Pleistozäns auf. Obwohl der Aufstieg und die Randsenkenbildung bis ins Pliozän sehr intensiv ist, geht die Tieflage des glaziären Pleistozäns über den Strukturtop, den Scheitelgraben und die sekundäre Randsenke hinweg. Vielleicht wurde der normale Aufstieg des Diapirs wegen zu großer Auflast für einige Zeit unterbrochen.

\section{Alter der Rinnen}

Für die Rinnen im Raum zwischen Elbmündung-Heide-Rendsburg-Preetz-Lauenburg-Wedel können durch die teilweise Füllung mit Lauenburger Ton und Holstein(bzw. Stör-)Interglazial eine elster- bzw. mindeleiszeitliche Entstehungszeit angenommen werden. Die Verbreitung des Lauenburger Tons scheint weitgehend durch das Vorhandensein glaziärer Rinnen als fjord-artige Talungen begünstigt oder verursacht zu sein. Rinnen mit Vorkommen von Lauenburger Ton lassen sich $\mathrm{zu}$ einem verbundenen System vereinigen. Durch spätere Vereisungen ist die ursprüngliche Rinnenfüllung z. T. wieder aus- 
geräumt worden, Rinnen sind erweitert oder neu gebildet worden. Hierdurch kommt es zu sehr starken Unterschieden in der Rinnenfüllung (Kiese, Sande, Geschiebemergel, Bekkensedimente, Lauenburger Ton, Holstein-Interglazial, dieses z. T. auch neben den Rinnen).

Wegen des ungeschützt ohne Moränen frei zu Tage liegenden Präglazials beim ersten Inlandeisvorstoß in der Elster-Vereisung wird während dieser ersten großen Vereisung die Rinnenbildung am intensivsten gewesen sein. Lediglich für die Rinnen an der Flensburger Innenförde, hinteren Schlei, inneren Eckernförder Bucht, inneren Kieler Förde und an der Oldenburger Mulde erscheint ein weichseleiszeitliches Alter wahrscheinlicher, weil die Innenteile, wenn auch nicht die äußeren Abschnitte, der Förden durch glaziäre Rinnen vorgezeichnet sind.

\section{Schriftenverzeichnis}

Hinsch, W. (1975): Präquartärer Untergrund und glaziäre Rinnen in Südostholstein. - Mitt. geol.-paläont. Inst. Univ. Hamburg, 44: 383-402; Hamburg.

Johannsen, A. \& Nachtigall, K. H. (1972): Geologisch-hydrogeologische Untersuchungen im Südteil des Kreises Herzogtum Lauenburg. - Meyniana, 22: 71-84; Kiel.

Jaritz, W. (1973): Zur Entstehung der Salzstrukturen Nordwestdeutschlands. - Geol. Jb., A 10: 77 S.; Hannover.

Косн, E. (1924): Die prädiluviale Auflagerungsfläche unter Hamburg und Umgebung. - Mitt. min.-geol. Staatsinst. Hamburg, 6: 29-96; Hamburg.

Kuster, H. \& Meyer, K.-D. (1979): Glaziäre Rinnen im mittleren und nordöstlichen Niedersachsen. - Eiszeitalter u. Gegenwart, 29: 135-156; Hannover.

LöHNERT, E. (1966): Glaziäre Rinnen im Raum Hamburg und ihre Beziehungen zum präquartären Untergrund. - Abh. naturwiss. Ver. Hamburg, N. F., 10, 47-51; Hamburg.

Rasmussen, L. B. (1966): Molluscan Faunas and Biostratigraphy of the Marine Younger Miocene Formations in Denmark. - Danmarks Geol. Unders., II, 88: 358 S., København.

\section{$\mathrm{K}$ arten}

HrNsCH, W. (1977a): Karte des präquartären Untergrundes in Schleswig-Holstein $1: 250000$. — Geol. LA. Schleswig-Holstein, Kiel.

- (1977b): Basis des glaziären Pleistozäns in Schleswig-Holstein 1:250000. - Geol. LA Schleswig-Holstein, Kiel. 